\title{
Differential Effects of Intravenous Anesthetics on PDGF-BB-Induced Vascular Smooth Muscle Cell Migration
}

\author{
Miki Iida ${ }^{a}$ Kumiko Tanabe ${ }^{a}$ Osamu Kozawa ${ }^{b}$ Hiroki Iida ${ }^{a}$

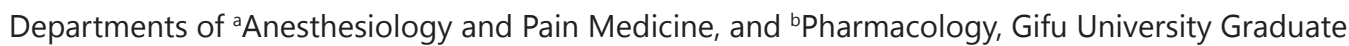 \\ School of Medicine, Gifu, Japan
}

\author{
Key Words \\ Propofol $\cdot$ Midazolam $\cdot$ Ketamine $\cdot$ Dexmedetomidine $\cdot$ Migration $・$ PDGF-BB $・$ Vascular \\ smooth muscle cell
}

\begin{abstract}
Background: Intravenous anesthetics are used during the perioperative and/or postoperative period in critically ill patients. Vascular smooth muscle cells (VSMCs) play important roles in vascular injury repair or restenosis after intervention. We previously reported that plateletderived growth factor (PDGF)-BB induces VSMC migration via extracellular signal-regulated kinase (ERK) and Akt in a VSMC line, A10 cells. In the present study, we investigated the effects of intravenous anesthetics on PDGF-BB-induced VSMC migration and the mechanism. Methods: VSMCs migration was assessed using Boyden chamber, and phosphorylation of each protein kinase was analyzed by Western blotting. Results: Propofol or midazolam but not ketamine or dexmedetomidine suppressed PDGF-BB-induced A10 cells migration in a concentration-dependent manner. The suppressive effects on migration were observed also in human aortic smooth muscle cells. Propofol or midazolam did not affect phosphorylation of PDGF receptor $\beta$ in A10 cells. Propofol or midazolam failed to affect PDGF-BB-induced phosphorylation of ERK or Akt. On the other hand, propofol or midazolam attenuated PDGFBB-induced phosphorylation of p38 mitogen-activated protein kinase (MAPK), but did not affect phosphorylation of stress-activated protein kinase/c-Jun $\mathrm{N}$-terminal kinase. Both ketamine and dexmedetomidine had no effect on the phosphorylation of p38 MAPK induced by PDGF-BB. Conclusion: These results strongly suggest that propofol or midazolam inhibits VSMC migration by PDGF-BB via suppression of p38 MAPK activation. Propofol or midazolam may affect VSMC function in critically ill patients.
\end{abstract}




\section{Introduction}

Intravenous anesthetics have been widely used in general anesthesia and for sedation in intensive care unit (ICU). The most common drugs employed for sedation in the ICU are the $\gamma$-aminobutyric acid (GABA) agonists' propofol and benzodiazepines, such as midazolam or diazepam. In addition, the use of the $\alpha_{2}$ agonist, dexmedetomidine, is currently increasing [1]. Ketamine is other agent that has been used for many years in the ICU setting for sedation, considered adjunctive therapy recently [1]. Intravenous anesthetics are firmly established to have direct effects on vascular smooth muscle cells (VSMCs), resulting in modulation of blood pressure. For example, ketamine or midazolam reduces both intracellular $\mathrm{Ca}^{2+}$

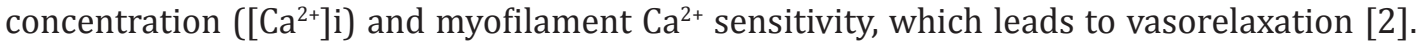
Propofol reduces $\left[\mathrm{Ca}^{2+}\right] \mathrm{i}$ via the protein kinase $\mathrm{C}(\mathrm{PKC})$-dependent pathway, increasing guanosine 3', 5'-cyclic monophosphate (cGMP) or hyperpolarization of $\mathrm{K}^{+}$channel [2]. In our previous study [3], we have demonstrated that propofol inhibits endothelin-1-induced $\mathrm{Ca}^{2+}$ influx and phosphoinositide-hydrolysis in VSMCs and the suppressive effect is exerted the point between the receptors and heterotrimeric GTP-binding protein. Higher concentration of dexmedetomidine contributes to vasoconstriction due to direct activation of its specific receptor on VSMCs [4].

Along with blood pressure modulation, VSMCs play important roles in vascular injury repair, angiogenesis, atherosclerosis [5], restenosis after angioplasty or by-pass graft surgery [6] and would healings [7]. VSMC migration and proliferation are major processes of these phenomena [5-7]. It has been reported that ketamine, but not propofol, inhibits epidermal growth factor (EGF)-induced human VSMC proliferation via the PKC pathway [8]. It has been reported that VSMC migration is regulated by a variety of growth factors, including platelet-derived-growth-factor (PDGF), basic fibroblast growth factor (bFGF), transforming growth factor- $\beta$ (TGF- $\beta$ ), EGF and insulin-like growth factor (IGF) [6]. Among them, PDGF is the strongest chemoattractant for VSMC migration [9]. VSMC migration following vascular injury is dependent on PDGF release [10]. It is generally known that PDGF is secreted by not only platelets, but also monocyte/macrophages, vascular endothelial cells and VSMCs [11]. PDGF expression is dynamic and responsive to variety of stimuli, including hypoxia, thrombin, cytokines, and growth factors [12]. Regarding the intracellular signaling, it has been reported that extracellular signal-regulated protein kinase (ERK), stress-activated protein kinase/c-Jun N-terminal kinase (SAPK/JNK), p38 mitogen-activated protein kinase (MAPK), phosphatidylinositol 3-kinase (PI3K) and PKC are involved in PDGF-BB-induced rat aortic VSMC migration $[13,14]$. In a rat VSMC line, A10 cells, we have demonstrated that PDGF-BB induces VSMC migration via the ERK pathway and the PI3K/Akt pathway [15]. As for the effects of anesthetics on VSMC migration, previous study using primary rat VSMCs showed that dexmedetomidine alone increases cell migration [16]. However, the effects of intravenous anesthetics on VSMC migration are not precisely clarified.

In the present study, we investigated whether intravenous anesthetics affect PDGF-BBinduced VSMC migration and the detailed mechanism. We herein demonstrate that propofol and midazolam among intravenous anesthetics inhibit PDGF-BB-induced VSMC migration via suppression of p38 MAPK activation.

\section{Materials and Methods}

\section{Materials}

PDGF-BB was obtained from R\&D Systems, Inc. (Minneapolis, MN). Propofol, midazolam and ketamine were purchased from Sigma Chemical Co. (St. Louis, MO). Dexmedetomidine was kindly provided by Orion Pharma (Turku, Finland). Antibodies against phosho-specific PDGF receptor $\beta$ (PDGFR $\beta$ ), phospho-specific Akt (Thr-308), phospho-specific Akt (Ser-473), Akt, phospho-specific ERK, ERK, phospho-specific SAPK/JNK, SAPK/JNK, phospho-specific p38 MAPK and p38 MAPK and were obtained from Cell Signaling Technology, Inc. (Beverly, MA). Antibody against GAPDH was purchased from Santa Cruz Biotechnology, Inc (Santa Cruz, 
CA). ECL Western blotting detection system was purchased from GE Healthcare (Buckinghamshire, UK). Other materials and chemicals were obtained from commercial sources. Propofol was dissolved in ethanol. The maximum concentration of ethanol was $0.1 \%$, which did not affect the cell migration assay or Western blot analysis.

\section{Cell culture and treatments}

Fetal rat aortic smooth muscle-derived A10 cells were obtained from American Type Culture Collection (Rockville, MD). A10 cells were seeded into 90-mm $\left(4 \times 10^{5}\right.$ cells/dish) diameter dishes and maintained in Dulbecco's modified Eagle's medium (DMEM) containing 10\% fetal bovine serum (FBS) at $37^{\circ} \mathrm{C}$ under a humidified atmosphere of $5 \%$ carbon dioxide and $95 \%$ air. After 6 days, the medium was exchanged for serum-free DMEM. The A10 cells were then used for Western blot analysis after $24 \mathrm{~h}$. The cells were pretreated with propofol, midazolam, ketamine or dexmedetomidine for $60 \mathrm{~min}$ before PDGF-BB stimulation when indicated. Primary cultured human aortic smooth muscle cells (HASMCs) were obtained from Cell Systems (Kirkland, WA). HASMCs were seeded into dishes coated with Attachment Factor ${ }^{\text {TM }}$ (Cell Systems, Kirkland, WA) and maintained in CSC Complete Medium (Cell Systems, Kirkland, WA) containing $10 \% \mathrm{FBS}$ at $37^{\circ} \mathrm{C}$ under a humidified atmosphere of $5 \%$ carbon dioxide and $95 \%$ air.

\section{Cell migration assay}

Cell migration was assessed in using Boyden chamber (polycarbonate membrane with 8- $\mu \mathrm{m}$ pores, Transwell ${ }^{\mathrm{TM}}$, Coring Costar Corp., Cambridge, MA). The upper chambers were coated with Attachment Factor $^{\mathrm{TM}}$ for HASMCs. The cells were trypsinized, and seeded $\left(3 \times 10^{4}\right.$ cells/well) onto the upper chamber in serum-free medium. The cells were pretreated with propofol, midazolam, ketamine or dexmedetomidine in lower chamber for $60 \mathrm{~min}$ at $37^{\circ} \mathrm{C}$. Then PDGF-BB was added to lower chamber. A10 cells were incubated for $9 \mathrm{~h}$ at $37^{\circ} \mathrm{C}$ with PDGF-BB. HASMCs were incubated with PDGF-BB for $24 \mathrm{~h}$ at $37^{\circ} \mathrm{C}$. The cells on the upper surface of the membrane were mechanically removed. The migrated cells adherent to the underside of the membrane were fixed with $4 \%$ paraformaldehyde and stained with 4,6-diamidino-2-phenylindole (DAPI) solution. The migrated cells were photographed and counted using fluorescent microscopy at a magnification of $20 \times$ by counting the stained cells from three randomly chosen high power fields.

\section{Western blot analysis}

The cultured A10 cells were stimulated by $30 \mathrm{ng} / \mathrm{ml}$ PDGF-BB in serum-free DMEM for $10 \mathrm{~min}$. The cells were washed twice with phosphate-buffered saline, and then lysed and sonicated in a lysis buffer containing $62.5 \mathrm{mM}$ Tris/ $\mathrm{HCl}$ (pH 6.8), $2 \%$ sodium dodecyl sulfate (SDS), $50 \mathrm{mM}$ dithiothreitol and $10 \%$ glycerol. The sample was subjected to SDS-polyacrylamide gel electrophoresis (PAGE) that was performed using by the method described by Laemmli [17]. A Western blot analysis was performed using phosphospecific PDGFR $\beta$ antibodies, GAPDH antibodies phospho-specific Akt (Thr-308) antibodies, phosphospecific Akt (Ser-473) antibodies, Akt antibodies, phospho-specific ERK antibodies, ERK antibodies, phospho-specific SAPK/JNK antibodies, SAPK/JNK antibodies, phospho-specific p38 MAPK antibodies or p38 MAPK antibodies with peroxidase-labeled anti-rabbit IgG antibodies used as secondary antibodies. Peroxidase activity on polyvinylidene fluoride membrane was visualized on X-ray film by means of an ECL Western blotting detection system.

\section{Statistical analysis}

The data were analyzed by ANOVA followed by Bonferroni's method for multiple comparisons between pairs. Values of $P<0.05$ were considered to be statistically significant. The data are expressed as the means \pm SD of triplicate determinations from three independent cell preparations. Each experiment was repeated three times with similar results.

\section{Results}

Effects of anesthetics on PDGF-BB-induced A10 cell migration

It has been reported that PDGF-BB induces migration of vascular smooth muscle A10 cells $[18,19]$. We previously confirmed that when the cells were exposed to PDGF-BB, A10 cells significantly migrated, compared with unstimulated A10 cells in a time-dependent 


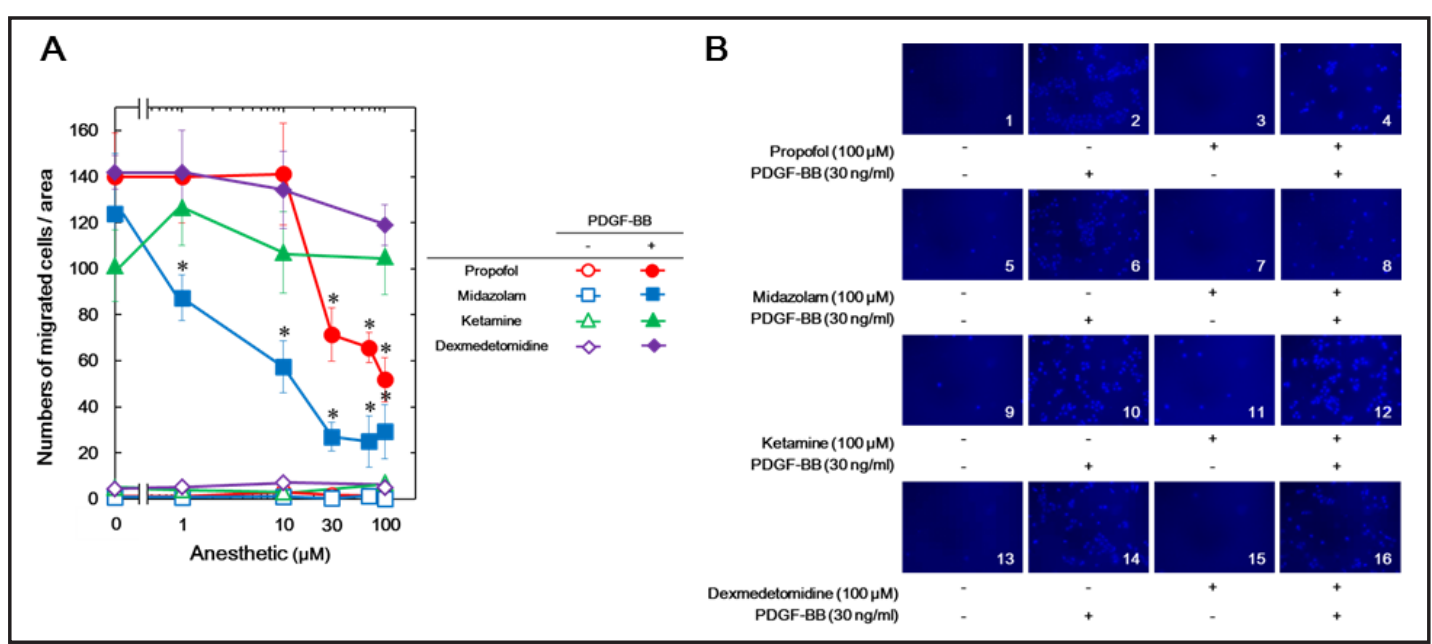

Fig. 1. Effects of anesthetics on PDGF-BB-induced migration of A10 cells. (A) The cells were pretreated with various concentrations of propofol (circles), midazolam (squares), ketamine (triangles) or dexmedetomidine (lozenges) for $1 \mathrm{~h}$, and then stimulated by $30 \mathrm{ng} / \mathrm{ml}$ PDGF-BB (closed symbols) or vehicle (open symbols) for $9 \mathrm{~h}$. The cells were then fixed and stained as described in Materials and methods. The average number of migrated cells from 3 randomly chosen fields that were migrated to the lower surface of the membrane was counted. Each value represents the mean \pm SD of triplicate independent determinations of a representative experiment carried out three times. Similar results were obtained with two additional and different cell preparations. ${ }^{*} P<0.05$, compared to the value of PDGF-BB alone. (B) Fluorescence microscopy of the migrated cells. The cells were pretreated with $100 \mu \mathrm{M}$ propofol (panels 3, 4), $100 \mu \mathrm{M}$ midazolam (panels 7, 8), $100 \mu \mathrm{M}$ ketamine (panels 11,12) or $100 \mu \mathrm{M}$ dexmedetomidine (panels 15, 16) or vehicle (panels $1,2,5,6,9,10,13,14$ ) for $1 \mathrm{~h}$, and then stimulated by $30 \mathrm{ng} / \mathrm{ml}$ PDGF-BB (panels 2,4,6,8,10,12,14,16) or vehicle (panels 1,3,5,7,9,11,13,15) for $9 \mathrm{~h}$. Panels show the cells which were migrated to the lower surface of the membrane. The cells were photographed by fluorescent microscopy at a magnification of $20 \times$.

Fig. 2. Effects of anesthetics on PDGF-BB-induced migration of HASMCs. The cells were pretreated with various concentrations of propofol (circles), midazolam (squares), ketamine (triangles) or dexmedetomidine (lozenges) for $1 \mathrm{~h}$, and then stimulated by $30 \mathrm{ng} / \mathrm{ml}$ PDGF-BB (closed symbols) or vehicle (open symbols) for 24 $h$. The cells were then fixed and stained as described in Materials and methods. The average number of migrated cells from

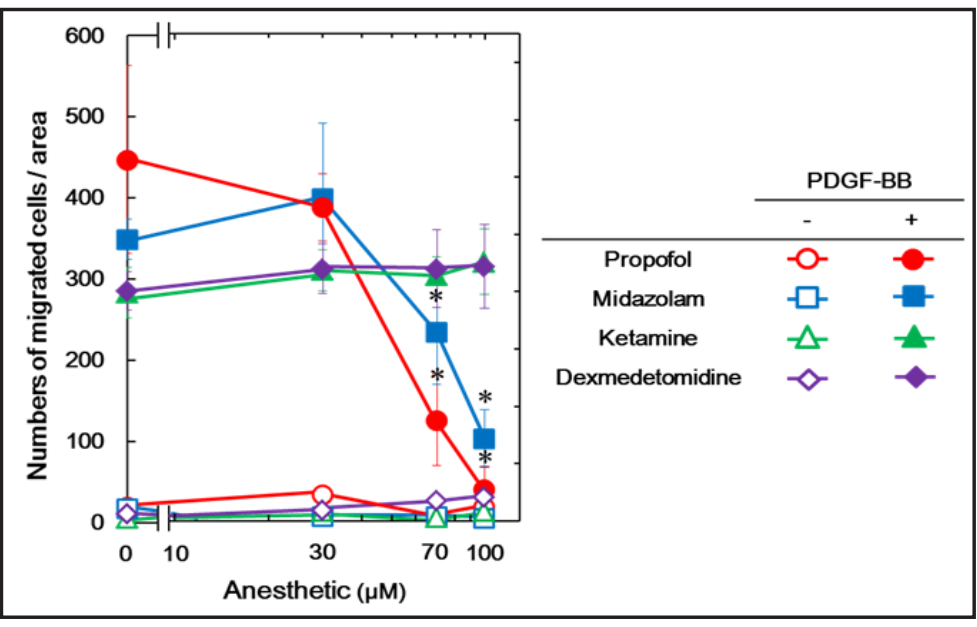

3 randomly chosen fields that were migrated to the lower surface of the membrane was counted. Each value represents the mean \pm SD of triplicate independent determinations of a representative experiment carried out three times. Similar results were obtained with two additional and different cell preparations. ${ }^{*} P<0.05$, compared to the value of PDGF-BB alone.

manner [15]. The stimulatory effect of PDGF-BB was observed at $6 \mathrm{~h}$, increased up to 12 $h$, and decreased thereafter [15]. To investigate the effects of anesthetics on PDGF-BBinduced VSMC migration, we examined the effect of propofol, midazolam, ketamine or 
Fig. 3. Effects of propofol or midazolam on PDGFBB-induced phosphorylation of PDGFR $\beta$ in A10 cells. The cultured cells were pretreated with 100 $\mu \mathrm{M}$ of propofol (A) or midazolam (B) for $1 \mathrm{~h}$, and then stimulated by $30 \mathrm{ng} / \mathrm{ml}$ PDGF-BB for $10 \mathrm{~min}$. The extracts of cells were subjected to SDS-PAGE with subsequent Western blot analysis with antibodies against phospho-specific PDGFR $\beta$ or GAPDH. Similar results were obtained with two additional and different cell preparations.

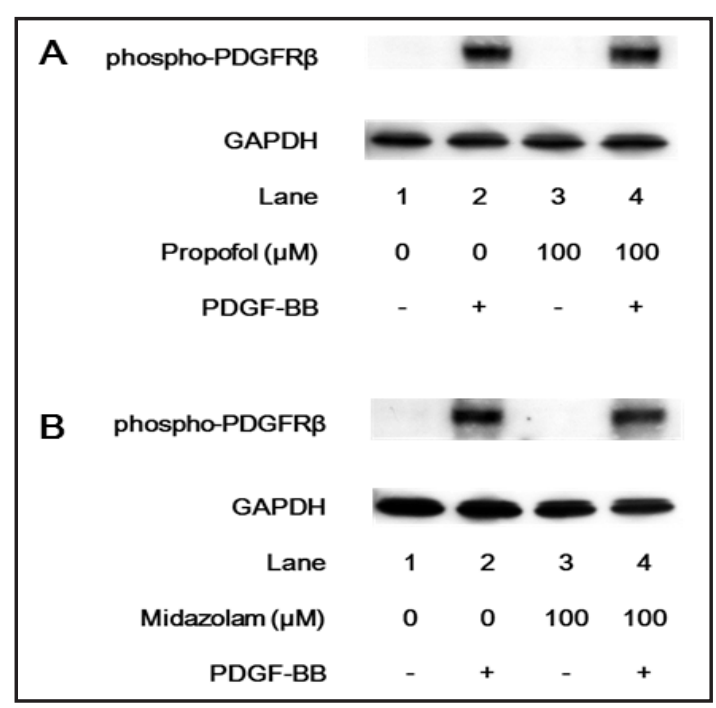

Fig. 4. Effects of propofol or midazolam on PDGFBB-induced phosphorylation of Akt in A10 cells. The cultured cells were pretreated with various concentrations of propofol (A) or midazolam (B) for $1 \mathrm{~h}$, and then stimulated by $30 \mathrm{ng} / \mathrm{ml}$ PDGF-BB for $10 \mathrm{~min}$. The extracts of cells were subjected to SDS-PAGE with subsequent Western blot analysis with antibodies against phospho-specific Akt (Thr-308), phospho-specific Akt (Ser-473) or Akt. Similar results were obtained with two additional and different cell preparations.

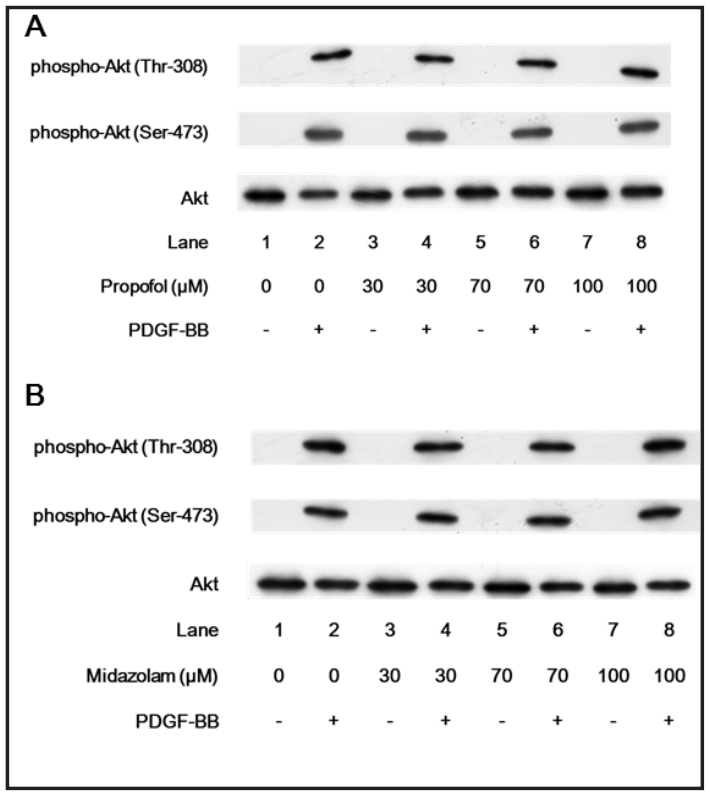

dexmedetomidine, widely used intravenous anesthetics, on PDGF-BB-induced A10 cell migration. Propofol or midazolam, which alone did not affect the cell migration, significantly suppressed PDGF-BB-induced A10 cell migration (Fig. 1A and 1B). The suppressive effect of propofol or midazolam was concentration-dependent between 30 and $100 \mu \mathrm{M}$, or 1 and 100 $\mu \mathrm{M}$, respectively (Fig. 1A). On the contrary, ketamine or dexmedetomidine failed to affect PDGF-BB-induced A10 cell migration up to $100 \mu \mathrm{M}$ (Fig. 1).

\section{Effects of anesthetics on PDGF-BB-induced HASMC migration}

In order to clarify whether propofol or midazolam suppresses PDGF-BB-induced cell migration in primary cultured HASMCs, we examined the effects of propofol, midazolam, ketamine or dexmedetomidine on PDGF-BB-induced HASMC migration. We found that the stimulatory effect of PDGF-BB on HASMC migration was observed at $12 \mathrm{~h}$ and increased up to $24 \mathrm{~h}$ (data not shown). Propofol or midazolam, which alone did not affect the cell migration, significantly suppressed the PDGF-BB-induced migration also in these cells (Fig. 2 ). On the contrary, ketamine or dexmedetomidine failed to affect the PDGF-BB-induced HASMC migration up to $100 \mu \mathrm{M}$ (Fig. 2). 


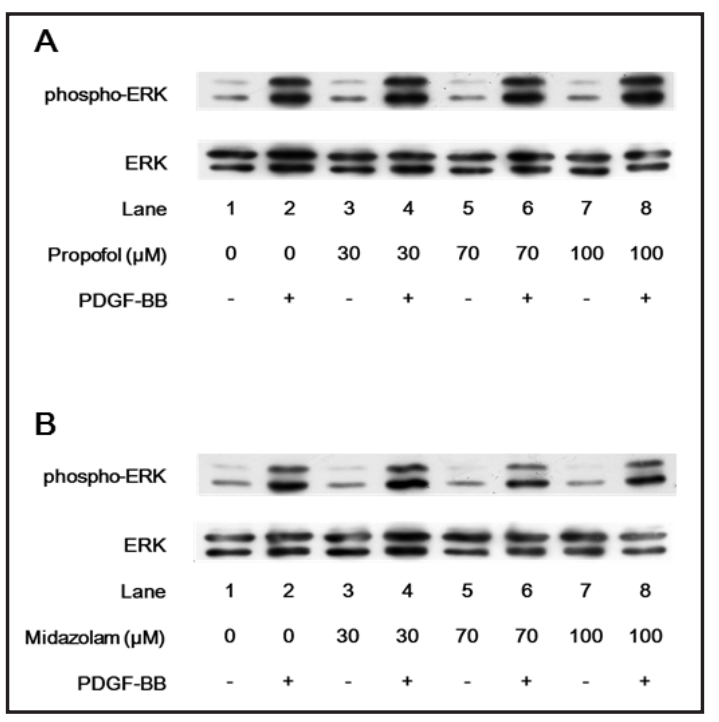

Fig. 5. Effects of propofol or midazolam on PDGFBB-induced phosphorylation of ERK in A10 cells. The cultured cells were pretreated with various concentrations of propofol (A) or midazolam (B) for $1 \mathrm{~h}$, and then stimulated by $30 \mathrm{ng} / \mathrm{ml}$ PDGF-BB for $10 \mathrm{~min}$. The extracts of cells were subjected to SDSPAGE with subsequent Western blot analysis with antibodies against phospho-specific ERK or ERK. Similar results were obtained with two additional and different cell preparations.

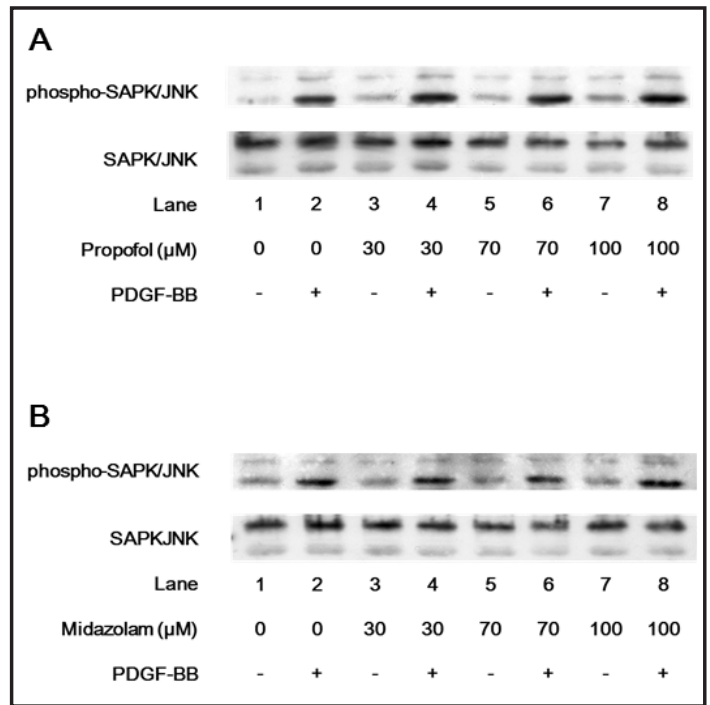

Fig. 6. Effects of propofol or midazolam on PDGFBB-induced phosphorylation of SAPK/JNK in A10 cells. The cultured cells were pretreated with various concentrations of propofol (A) or midazolam (B) for $1 \mathrm{~h}$, and then stimulated by $30 \mathrm{ng} / \mathrm{ml}$ PDGF-BB for $10 \mathrm{~min}$. The extracts of cells were subjected to SDS-PAGE with subsequent Western blot analysis with antibodies against phospho-specific SAPK/JNK or SAPK/JNK. Similar results were obtained with two additional and different cell preparations.

Effects of propofol or midazolam on PDGF-BB-induced phosphorylation of PDGFR in A10 cells

Next, we investigated the mechanism how propofol or midazolam affects PDGF-BBinduced A10 cell migration. It has been reported that there are two PDGFR proteins, PDGFR $\alpha$ and PDGFR $\beta$, with PDGFR $\alpha$ able to bind PDGF A-chain and B-chain, and PDGFR $\beta$ able to bind only B-chain [7]. PDGF-BB induces activation of PDGFR $\beta$ for working out mechanisms of signal transduction downstream of receptors [7]. In order to investigate whether propofol or midazolam acts on PDGFR in A10 cells, we examined the effect of these anesthetics on PDGFBB-induced phosphorylation of PDGFR $\beta$. We found that PDGF-BB-induced phosphorylation of PDGFR $\beta$ was not affected by propofol or midazolam up to $100 \mu \mathrm{M}$ in A10 cells (Fig. 3A and 3B).

Effects of propofol or midazolam on PDGF-BB-induced phosphorylation of Akt or ERK in A10 cells

We have previously shown that PDGF-BB stimulates A10 cell migration at least in part via the ERK pathway and the PI3K/Akt pathway [15]. Therefore, in order to explore whether the ERK pathway and/or PI3K/Akt pathway are involved in the inhibitory effect of propofol or midazolam on PDGF-BB-induced A10 cell migration, we examined the effect of propofol or midazolam on PDGF-BB-induced phosphorylation of ERK or Akt. However, we found that PDGF-BB-induced phosphorylation of Akt at Thr-308 and Ser-473 residues (Fig. 4) or ERK (Fig. 5) were not affected by propofol or midazolam up to $100 \mu \mathrm{M}$ in A10 cells. 
Fig. 7. Effects of propofol, midazolam, ketamine or dexmedetomidine on PDGF-BBinduced phosphorylation of p38 MAPK in A10 cells. The cultured cells were pretreated with various concentrations of propofol (A), midazolam (B), ketamine (C) or dexmedetomidine (D) for $1 \mathrm{~h}$, and then stimulated by $30 \mathrm{ng} / \mathrm{ml}$ PDGF-BB for $10 \mathrm{~min}$. The extracts of cells were subjected to SDS-PAGE with subsequent Western blot analysis with antibodies against phospho-specific p38 MAPK or p38 MAPK. Similar results were obtained with two additional and different cell preparations.

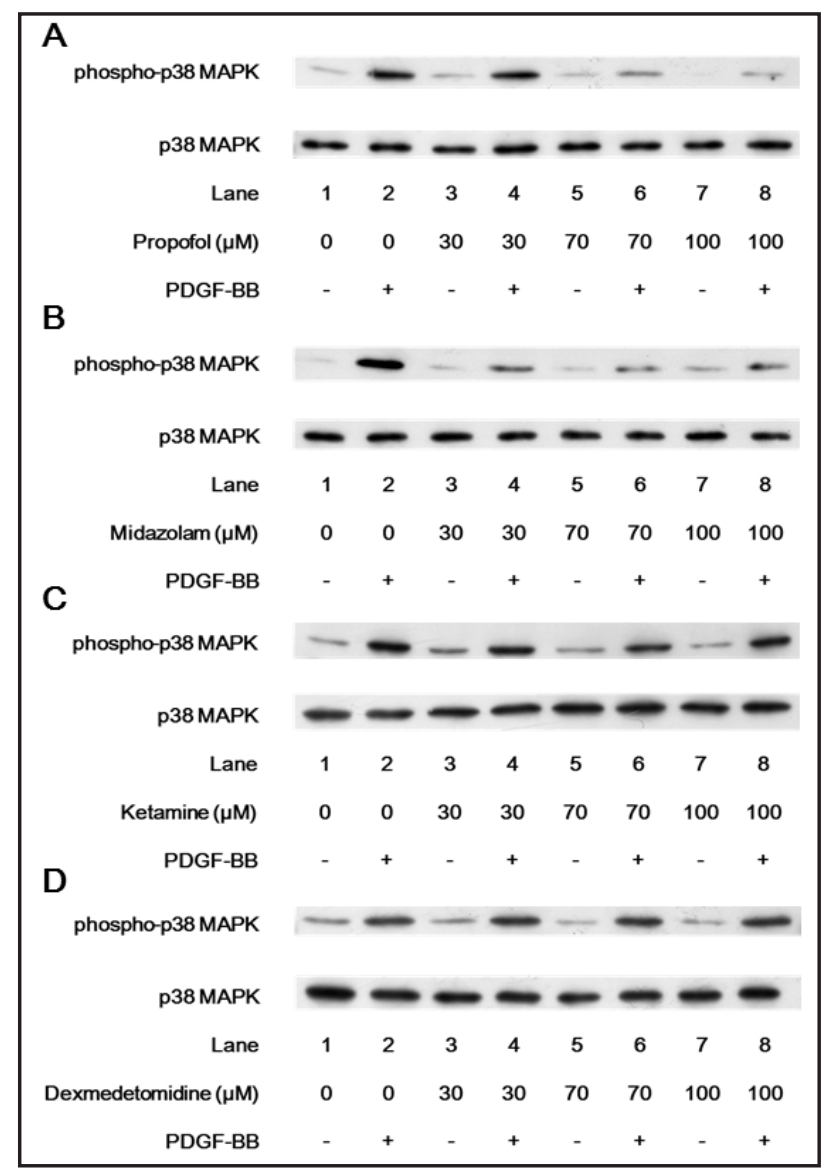

Effects of propofol or midazolam on PDGF-BB-induced phosphorylation of SAPK/JNK or p38 MAPK in A10 cells

In addition to the ERK pathway and the PI3K/Akt pathway, it has been demonstrated that SAPK/JNK and p38 MAPK, members of the MAPK superfamily, play as positive regulators in PDGF-BB-induced VSMC migration [14]. We confirmed that PDGF-BB time dependently induced phosphorylation of SAPK/JNK and p38 MAPK in A10 cells. The PDGFBB-induced phosphorylation of both SAPK/JNK and p38 MAPK reached the peak at 10 min (data not shown). In addition, we found that SB203580, a specific p38 MAPK inhibitor [20], or SP600125, a specific SAPK/JNK inhibitor [21], suppressed PDGF-BB-induced A10 cell migration (data not shown). In order to investigate whether the SAPK/JNK and/or p38 MAPK are involved in the inhibitory effect of propofol or midazolam on PDGF-BB-induced A10 cell migration, we examined the effect of propofol or midazolam on PDGF-BB-induced phosphorylation of SAPK/JNK or p38 MAPK. Both propofol and midazolam did not influence PDGF-BB-induced phosphorylation of SAPK/JNK up to $100 \mu \mathrm{M}$ in A10 cells (Fig. 6). On the contrary, both propofol and midazolam remarkably suppressed PDGF-BB-induced p38 MAPK phosphorylation in A10 cells (Fig. 7A and 7B). The suppressive effect of propofol or midazolam was observed even at $70 \mu \mathrm{M}$ or $30 \mu \mathrm{M}$, respectively.

Effects of ketamine or dexmedetomidine on PDGF-BB-induced phosphorylation of p38 MAPK in A10 cells

In the present study, our findings suggest that the suppressive effect of propofol or midazolam in PDGF-BB-induced A10 cell migration is mediated by the inhibition of p38 MAPK activation. Therefore, we investigated whether ketamine or dexmedetoidine suppresses PDGF-BB-induced phosphorylation of p38 MAPK in A10 cells. We found that both ketamine and dexmedetomidine failed to attenuate PDGF-BB-induced phosphorylation of p38 MAPK up to $100 \mu \mathrm{M}$ (Fig. 7C and 7D). 


\section{Discussion}

In the present study, we first demonstrated that propofol or midazolam significantly suppressed PDGF-BB-induced migration in vascular smooth muscle A10 cells, in a concentration-dependent manner. On the contrary, ketamine and dexmedetomidine did not affect A10 cell migration. Additionally, we found that these effects of anesthetics were observed also in HASMCs, a different vascular smooth muscle cell type. Therefore, it is likely that the anesthetic-effects on cell migration are common in VSMC. To date, there is only one report regarding the effect of intravenous anesthetics on VSMC migration, showing that dexmedetomidine by itself induces VSMC migration as far as we know [16]. To the best of our knowledge, this is probably the first report of the inhibitory effect of intravenous anesthetics on VSMC migration.

We demonstrated that PDGF-BB-induced phosphorylation of PDGFR $\beta$ was not affected by propofol or midazolam. Then, we investigated the action point of propofol or midazolam in PDGF-BB-induced A10 cell migration. With regard to the intracellular signaling in VSMC, we previously demonstrated that PDGF-BB induces A10 cell migration via the ERK pathway and the PI3K/Akt pathway [15]. Therefore, we examined the effects of propofol or midazolam on PDGF-BB-induced phosphorylation of ERK and Akt. We found that PDGF-BBinduced phosphorylation of Akt at Thr-308 and Ser-473 residues, or ERK were not affected by propofol or midazolam up to $100 \mu \mathrm{M}$ in A10 cells. Based on our findings, it seems unlikely that the anti-migratory effect of propofol or midazolam is involved in these pathways. The MAPK superfamily mediates intracellular signal transduction of variety of extracellular factors and plays a central role in cell function [22]. Three major mammalian MAPKs, ERK, p38 MAPK and SAPK/JNK, are well known to mediate intracellular signaling [22]. In addition to ERK, the SAPK/JNK or p38 MAPK pathway has been implicated in VSMC migration [14, 23]. Thus, we confirmed that PDGF-BB induced phosphorylation of SAPK/JNK or p38 MAPK in A10 cells, and that SB203580 or SP600125, a specific inhibitor of p38 MAPK or SAPK/ JNK, respectively, truly reduced the PDGF-BB-induced A10 cell migration. Based on these findings, it is probable that PDGF-BB promotes A10 cell migration via p38 MAPK and SAPK/ JNK as well as ERK and Akt. Next, in order to investigate whether p38 MAPK and/or SAPK/ JNK are involved in inhibitory effects of propofol or midazolam on PDGF-BB-induced A10 cell migration, we examined the effect of propofol or midazolam on phosphorylation of PDGF-BBinduced p38 MAPK or SAPK/JNK. We showed that both propofol and midazolam attenuated PDGF-BB-induced phosphorylation of p38 MAPK, but not SAPK/JNK. We confirmed that both ketamine and dexmedetomidine failed to affect PDGF-BB-induced phosphorylation of p38 MAPK up to $100 \mu \mathrm{M}$. Taking these results into account, it is most likely that propofol or midazolam inhibits VSMC migration via suppression of p38 MAPK activation. The potential mechanism of the suppression by propofol or midazolam of PDGF-BB-induced VSMC migration shown herein is summarized in Fig. 8.

P38 MAPK is strongly activated by stress, including mechanical stretch, ultraviolet-light, oxidants, lipopolysaccharide, heat-shock and cytokines [24]. It has been reported that p38 MAPK plays a crucial role in vascular hypertrophy [25], and is important signal transducer mediating the stress-induced cell responses essential for VSMC migration and proliferation [24]. In addition, it has been shown that p38 MAPK activation is closely associated with VSMC apoptosis in response to mechanical stretch [24]. In the present study, we demonstrated that propofol or midazolam inhibited PDGF-BB-induced p38 MAPK phosphorylation in A10 cells, whereas ketamine and dexmedetomidine had no effect on the phosphorylation. However, the details behind the suppression by propofol or midazolam of the p38 MAPK pathway in VSMC remains obscure. Further investigations are necessary to clarify the exact mechanisms how propofol or midazolam affect VSMC migration.

We here showed that propofol or midazolam but not ketamine or dexmedetomidine suppressed PDGF-BB-induced VSMC migration. These anesthetics vary in their chemical structures and target receptors $[26,27]$. Ketamine is an $N$-methyl- $D$-aspartate receptor antagonist and dexmedetomidine is an $\alpha_{2}$ adrenoceptor agonist [1]. Both propofol and 
Fig. 8. Schematic representation of the potential mechanism of the suppression by propofol or midazolam of PDGF-BB-induced VSMC migration. PDGF-BB, platelet-derived growth factor-BB; ERK, extracellular signal-regulated protein kinase; SAPK/JNK, stress-activated protein kinase/c-Jun N-terminal kinase; p38 MAPK, p38 mitogen-activated protein kinase; VSMC, vascular smooth muscle cell.

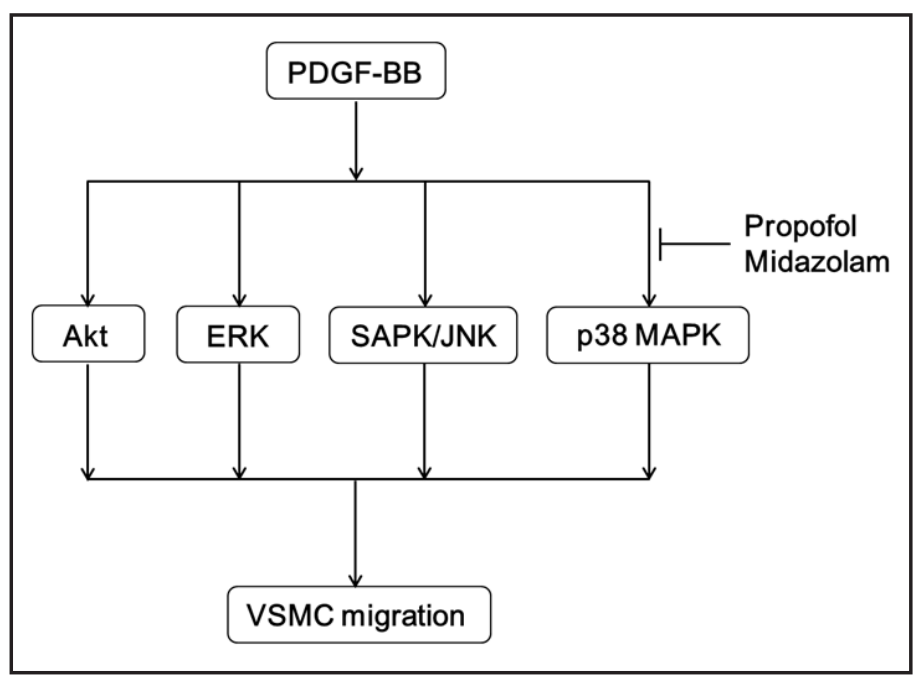

midazolam act on $\mathrm{GABA}_{\mathrm{A}}$ receptor [1]. Therefore, it seems likely that differential effects of these anesthetics on PDGF-BB-induced VSMC migration are related to variation of their target receptors.

It is generally established that a number of cytokines play a major role in the response to injury and infection and in development of organ damage in critically ill patients [28]. PDGF has been shown to stimulate the synthesis of tumor necrosis factor (TNF), which is proposed to be a key mediator of organ injury during sepsis, and in response to endotoxin [28]. Especially in septic patients, it has been reported that PDGF-BB levels in serum would be a useful laboratory marker to predict survival [29]. According to these opinions, critically ill patients are often exposed variety of stimuli which promote the release of PDGF-BB. It is recognized that PDGF is expressed at very low levels in normal vessels [9]. On the other hand, after injury, PDGF-BB is released from various cells in addition to platelets and induces VSMC migration $[9,30]$. VSMCs dramatically increase its rate of cell migration and proliferation, and play a pivotal role in vascular repair and wound healings [5, 30]. VSMC migration is also important event in the pathology of vascular disease such as atherosclerosis and restenosis after intervention [6]. Based on these findings, PDGF-induced VSMC migration promotes not only vascular injury repair, angiogenesis or wound healings but also development of atherosclerosis or restenosis after angioplasty or by-pass graft surgery. Therefore, it might depend on the patient's situations whether inhibition of VSMC migration is advantageous or not. That is to say, there is a possibility that inhibition of VSMC migration is preventing atherosclerosis and restenosis after intervention. On the contrary, it is likely that inhibition of VSMC migration is harmful to the patient after surgical procedure or trauma subsequently to delay wound healings.

In the clinical situations, it has been demonstrated that the plasma concentration of propofol or midazolam in ICU is approximately $4.0-7.5 \mu \mathrm{M}$ or $0.8-1.5 \mu \mathrm{M}$, respectively [31]. The plasma concentration of ketamine at $5 \mathrm{~min}$ after the intravenous administration is reportedly approximately 10-60 $\mu \mathrm{M}$ [8], and that clinically relevant plasma concentration of dexmedetomidine for sedation in humans is in the range 0.01-0.03 $\mu \mathrm{M}$ [32]. In the present study, we demonstrated that clinically used dose of midazolam, at least $1 \mu \mathrm{M}$, attenuated PDGF-BB-induced A10 cell migration. Thus, it is possible that the concentration of midazolam physiologically reaches that which promotes the effect shown here. On the other hand, the inhibitory effect of propofol on PDGF-BB-induced A10 cell migration was significantly observed over the range 30-100 $\mu \mathrm{M}$, which were higher than those used clinically. In many experiments using cultured VSMCs, higher concentrations of propofol than clinical use are needed for exerting various effects. For example, we previously reported that propofol attenuates the endothelin-1-induced $\mathrm{Ca}^{2+}$ influx only $100 \mu \mathrm{M}$, in A10 cells [3]. Therefore, it is possible that the difference of effective concentrations of propofol between clinical use 
and in vitro study is due to experimental conditions between in vivo and in vitro. Further investigations are necessary to clarify the details behind effect of propofol on VSMC functions.

In conclusion, our findings strongly suggest that propofol or midazolam, but not ketamine or dexmedetomidine, inhibits VSMC migration by PDGF-BB via suppression of $\mathrm{p} 38$ MAPK activation.

\section{Disclosure Statement}

All authors declare no conflict of interest.

\section{Acknowledgements}

This work was supported in part by a Grant-in-Aid for Scientific Research (No. 23592247) from the Ministry of Education, Science, Sports and Culture of Japan. We are very grateful to Yumiko Kurokawa, technical assistant of Department of Pharmacology, Gifu University Graduate School of Medicine, for her skillful technical assistance.

\section{References}

1 McGrane S, Pandharipande PP: Sedation in the intensive care unit. Minerva Anestesiol 2012;78:369-380.

-2 Akata T: General anesthetics and vascular smooth muscle: Direct actions of general anesthetics on cellular mechanisms regulating vascular tone. Anesthesiology 2007;106:365-391.

3 Tanabe K, Kozawa O, Kaida T, Matsuno H, Niwa M, Ohta S, Dohi S, Uematsu T: Inhibitory effects of propofol on intracellular signaling by endothelin-1 in aortic smooth muscle cells. Anesthesiology 1998;88:452-460.

-4 Seyrek M, Halici Z, Yildiz O, Ulusoy HB: Interaction between dexmedetomidine and $\alpha$-adrenergic receptors: Emphasis on vascular actions. J Cardiothorac Vasc Anesth 2011;25:856-862.

-5 Owens GK, Kumar MS, Wamhoff BR: Molecular regulation of vascular smooth muscle cell differentiation in development and disease. Physiol Rev 2004;84:767-801.

6 Willis AI, Pierre-Paul D, Sumpio BE, Gahtan V: Vascular smooth muscle cell migration: Current research and clinical implications. Vasc Endovascular Surg 2004;38:11-23.

7 Bowen-Pope DF, Raines EW: History of discovery: Platelet-derived growth factor. Arterioscler Thromb Vasc Biol 2011;31:2397-2401.

8 Shiga Y, Minami K, Segawa K, Uezono Y, Shiraishi M, Sata T, Yamamoto C, Sung-Teh K: The inhibition of aortic smooth muscle cell proliferation by the intravenous anesthetic ketamine. Anesth Analg 2004;99:1408-1412.

-9 Raines EW: PDGF and cardiovascular disease. Cytokine Growth Factor Rev 2004;15:237-254.

$\rightarrow 10$ Newby AC: An overview of the vascular response to injury: A tribute to the late Russell Ross. Toxicol Lett 2000;112-113:519-529.

11 Pierras K, Sjöblom T, Rubin K, Heldin CH, Östman A: PDGF receptors as cancer drug targets. Cancer Cell 2003;3:439-443.

12 Andrae J, Gallini R, Betsholtz C: Role of platelet-derived growth factors in physiology and medicine. Genes Dev 2008;22:1276-1312.

13 Pukac L, Huangpu J, Karnovsky MJ: Platelet-derived growth factor-BB, insulin-like growth factor-I, and phorbol ester activate different signaling pathway for stimulation of vascular smooth muscle cell migration. Exp Cell Res 1998;242:548-560.

14 Zhan Y, Kim S, Izumi Y, Izumiya Y, Nakao T, Miyazaki H, Iwao H: Role of JNK, p38, and ERK in plateletderived growth factor-induced vascular proliferation, migration, and gene expression. Arterioscler Thromb Vasc Biol 2003;23:795-801.

15 Iida M, Tanabe K, Matsushima-Nishiwaki R, Kozawa O, Iida H: Adenosine monophosphate-activated protein kinase regulates platelet-derived growth factor-BB-induced vascular smooth muscle cell migration. Arch Biochem Biophys 2013;530:83-92. 
16 Richman JG, Regan JW: $\alpha_{2}$-adrenergic receptors increase cell migration and decrease F-actin labeling in rat aortic smooth muscle cells. Am J Physiol Cell Physiol 1998;274:C654-C662.

17 Laemmli UK: Cleavage of structural proteins during the assembly of the head of bacteriophage T4. Nature 1970;5259:680-685.

18 Lo HM, Hung CF, Tseng YL, Chen BH, Jian JS, Wu WB: Lycopene binds PDGF-BB and inhibits PDGF-BBinduced intracellular signaling transduction pathway in rat smooth muscle cells. Biochem Pharmacol 2007;74:54-63.

19 Stec DE, Gannon KP, Beaird JS, Drummond HA: 20-Hydroxyeicosatetraenoic acid (20-HETE) stimulates migration of vascular smooth muscle cells. Cell Physiol Biochem 2007;19:121-128.

20 Cuenda A, Rouse J, Doza YN, Meier R, Cohen P, Gallagher TF, Young PR, Lee JC: SB 203580 is a specific inhibitor of a MAP kinase homologue which is stimulated by cellular stresses and interleukin-1. FEBS Lett 1995;364:229-233.

21 Bennett BL, Sasaki DT, Murray BW, O’Leary EC, Sakata ST, Xu W, Leisten JC, Motiwala A, Pierce S, Satoh Y, Bhagwat SS, Manning AM, Anderson DW: SP600125, an anthrapyrazolone inhibitor of Jun N-terminal kinase. Proc Natl Acad Sci U S A 2001;98:13681-13686.

22 Kyriakis JM, Avruch J: Mammalian mitogen-activated protein kinase signal transduction pathways activated by stress and inflammation. Physiol Rev 2001;81:807-869.

23 Ishizawa K, Izawa-Ishizawa Y, Ohnishi S, Motobayashi Y, Kawazoe K, Hamano S, Tsuchiya K, Tomita S, Minakuchi K, Tamaki T: Quercetin glucronide inhibits cell migration and proliferation by platelet-derived growth factor in vascular smooth muscle cells. J Pharmacol Sci 2009;109:257-264.

24 Li C, Xu Q: Mechanical stress-initiated signal transductions in vascular smooth muscle cells. Cell Signal 2000;12:435-445.

-25 Usio-Fulao M, Alexander RW, Akers M, Griendling KK: p38 mitogen-activated protein kinase is a critical component of the redox-sensitive signaling pathways activated by angiotensin II. J Biol Chem 1998;273:15022-15029.

26 Lugli AK, Yost CS, Kindler CH: Anaesthetic mechanisms: update on the challenge of unravelling the mystery of anaesthesia. Eur J Anaesthesiol 2009;26:807-820.

27 Ma D, Rajakumaraswamy N, Maze M: $\alpha 2$-adrenoceptor agonists: shedding light on neuroprotection?. Br Med Bull 2004;71:77-92.

28 Bellomo R: The cytokine network in the critically ill. Anaesth Intensive Care 1992;20:288-302.

29 Brueckmann M, Hoffmann U, Engelhardt C, Lang S, Fukudome K, Haase KK, Liebe V, Kaden JJ, Putensen C, Borggrefe M, Huhle G: Prognostic value of platelet-derived growth factor in patients with severe sepsis. Growth Factors 2007;25:15-24.

30 Barrientos S, Stojadinovic 0, Golinko MS, Brem H: Growth factors and cytokines in wound healing. Wound Repair Regen 2008;16:585-601.

- 31 Bjelland TW, Klepstad P, Haugen BO, Nilsen T, Dale O: Effects of hypothermia on the disposition of morphine, midazolam, fentanyl, and propofol in intensive care unit patients. Drug Metab Dispos 2013;41:214-223.

-32 Ebert TJ, Hall JE, Barney JA, Uhrich TD: The effects of increasing plasma concentrations of dexmedetomidine in humans. Anesthesiology 2000;93:382-394. 